\title{
DIVERSIDAD GENÉTICA EN BOVINOS DE OCHO REGIONES EN COSTA RICA ${ }^{1}$
}

\author{
Juan Miguel Cordero-Solórzano², Bernardo Vargas-Leitón ${ }^{3}$,Bernal León-Rodríguez ${ }^{2}$, Idania Chacón-González ${ }^{2}$, \\ Marco Martínez-Pichardo ${ }^{3}$
}

\section{RESUMEN}

Diversidad genética en bovinos de ocho regiones en Costa Rica. El objetivo del presente estudio fue explorar el grado de diversidad genética inter-regional presente en el ganado bovino de Costa Rica. Se colectaron 1498 muestras de ADN (año 2013) procedentes de ocho diferentes regiones del país. Se calcularon las frecuencias alélicas y los principales parámetros genéticos poblacionales para dieciocho marcadores microsatélite. Se realizó además un análisis de varianza molecular y se calcularon las distancias genéticas entre bovinos de diferentes regiones. A nivel nacional se observó un alto grado de diversidad, con un número promedio de 14,6 $\pm 1,01$ alelos observados y $5,6+0,37$ alelos efectivos por marcador. La heterocigosis observada (Ho) fue $0,76 \pm 0,01$ y la esperada (He) $0,81 \pm 0,01$. El contenido de información polimórfica (CIP) fue de $0,79 \pm 0,06$ y el índice de consanguinidad $\left(\mathrm{F}_{\mathrm{IS}}\right)$ fue de $0,06 \pm 0,004$. A nivel de regiones, la Ho varió desde $0,73 \pm 0,02$ en la región Central Sur hasta $0,78 \pm 0,01$ en la región Huetar Norte. El dendrograma mostró tres agrupaciones claramente diferenciadas, con las regiones Central Metropolitana y Central Occidental en un grupo; Huetar Caribe, Central Sur, Pacífico Central y Chorotega en un segundo grupo; y Huetar Norte y Brunca en un tercer grupo intermedio. Los estimados de diferenciación genética $\mathrm{R}_{\mathrm{ST}}$ fueron significativos entre regiones de distintos grupos y no significativos entre regiones de un mismo grupo. Las diferencias genéticas entre regiones se relacionaron con la proliferación diferenciada de tipos raciales en función de su adaptabilidad a las condiciones agroecológicas y a los sistemas de producción imperantes en cada región.

Palabras claves: frecuencias alélicas, distancia genética, marcador microsatélite.

\begin{abstract}
Genetic diversity in cattle of eight regions in Costa Rica. The aim of this study was to explore the extent of inter-regional genetic diversity present in the cattle of Costa Rica. 1498 DNA samples were collected (year 2013) from eight different regions within the country. Allelic frequencies and major population genetic parameters were determined for eighteen microsatellite markers. An analysis of molecular variance was also carried out and genetic distances were calculated between cattle from different regions. At the national level, a high allelic diversity was found, with an average of 14.6 \pm 1.01 observed alleles and 5.6+0.37 effective alleles per marker. Observed (Ho) and expected $(\mathrm{He})$ heterozygosities were $0.76 \pm 0.01$ and $0.81 \pm 01$, respectively. Polymorphic Information Content (PIC) and Coefficient of Inbreeding $\left(\mathrm{F}_{\text {IS }}\right)$ were $0.79 \pm 0.06$ and $0.06 \pm 0.004$, respectively. At the regional level, Ho ranged between $0.73 \pm 0.02$ in the South Central region to $0.78 \pm 0.01$ in the North Huetar region. The dendrogram showed three clearly distinct groups, Metropolitan Central and West Central regions in one group, Caribbean Huetar, South Central, Central Pacific and Chorotega regions in a second group; and North Huetar and Brunca regions in a third intermediate group. Estimates of genetic differentiation (RST) were significant between regions from different groups and non-significant for regions within the same group. Genetic differences between regions are related to differential proliferation of breed groups based on their adaptability to the agro-ecological conditions and production systems prevailing in each region.
\end{abstract}

Keywords: allelic frequencies, genetic distance, microsatellite marker.

1 Recibido: 17 setiembre, 2014. Aceptado: 18 de noviembre, 2014. Este estudio se realizó dentro del marco del proyecto de investigación SIA 0085-12, Universidad Nacional, financiado por la Comisión de Incentivos del Ministerio de Ciencia y Tecnología FI-370-11, Costa Rica.

2 Ministerio de Agricultura y Ganadería, Servicio Nacional de Salud Animal (SENASA), Laboratorio Nacional de Servicios Veterinarios (LANASEVE), Laboratorio de Bioseguridad (LSE). Campus Lagunilla, Barreal de Heredia, Costa Rica. Apdo Postal 3-3006 Heredia, Costa Rica.juanmcord@gmail.com, bleon@senasa.go.cr, ichacon@senasa.go.cr

3 Universidad Nacional de Costa Rica, Posgrado Regional en Ciencias Veterinarias, Tropicales. Campus Benjamín Núñez, Lagunilla Heredia. Apdo. Postal 304-3000 Heredia, Costa Rica. bernardo.vargas.leiton@una.cr, marco.medvet@yahoo.es 


\section{INTRODUCCIÓN}

Los primeros bovinos fueron traídos a Costa Rica a finales del siglo XVI, siendo principalmente ganado Bos taurus, originario de la península ibérica (Quirós, 2006), el cual fue introducido progresivamente en la región centroamericana. Este ganado estaba constituido por una mezcla de diversos tipos raciales ibéricos que localmente recibieron la denominación de criollo.

En la época contemporánea, las primeras importaciones de razas mejoradas fueron Devonshire y Durham, a mediados del siglo XVIII, las cuales procedían de Inglaterra (Quirós, 2006). El éxito obtenido en estos primeros mestizajes llevó a posteriores importaciones de otras razas. La primera de Holstein y Jersey sucedió en el año 1880 (Quirós, 2006). Las de Bos indicus se realizaron a principios del Siglo XX, procedentes de Jamaica (Quirós, 2006). De esta manera, durante las últimas décadas del siglo pasado se dio una sustitución paulatina del ganado criollo por razas Bos taurus o Bos indicus.

El tamaño de la población bovina ha sido bastante fluctuante; un censo realizado en 1891 indicaba un total de 345665 bovinos (Quirós, 2006). Posteriormente, la población bovina creció hasta principios de la década de los ochenta, donde alcanzó un máximo de 2,2 millones de cabezas. A finales del siglo pasado, la población se redujo hasta un total de 1358209 animales, con base en el último censo ganadero, y distribuidas en 38365 establecimientos de ganado bovino (CORFOGA, 2000), de los cuales un 60,6\% se dedicaban a la producción de carne, $16,7 \%$ a la lechería especializada y $22,7 \%$ al doble propósito.

En Costa Rica no se ha realizado hasta el momento ningún estudio sobre diversidad genética de ganado bovino a nivel nacional. Incluso la información sobre abundancia de diferentes tipos raciales es escasa, imprecisa y desactualizada. Según el último censo, Jersey es una de las razas más abundantes junto con la Holstein, Guernsey y Pardo Suizo (CORFOGA, 2000). Entre las razas cárnicas, la Brahman es predominante, seguida por la Simmental (CORFOGA, 2000). Sin embargo, la gran mayoría del ganado vacuno en el país, principalmente en sistemas de carne y doble propósito, es producto de múltiples cruces indeterminados entre diversas razas Bos taurus y/o Bos indicus.

Actualmente, con la disponibilidad de técnicas eficientes de análisis de ADN es posible medir con mayor precisión el grado de variabilidad genética presente en una población. La caracterización genética molecular se realiza fundamentalmente para explorar la diversidad genética dentro y entre distintas poblaciones animales, y para determinar relaciones genéticas entre ellas (FAO, 2010). Muchas de estas técnicas han sido utilizadas para el análisis de estructura y diversidad genética dentro y entre poblaciones bovinas (Lara et al., 2005; Cañón et al., 2008; Pizarro et al., 2009; Villalobos-Cortés et al., 2011; Acosta et al., 2013).

La toma de muestras para el análisis molecular se puede combinar con las encuestas y/o el seguimiento, ya que la información molecular por sí sola no se puede usar para tomar decisiones sobre utilización y conservación (FAO, 2010). De esta manera, los estudios genéticos pueden contribuir a identificar subgrupos animales que podrían ser importantes para futuros programas de gestión de recursos genéticos.

La mayoría de los estudios utilizan marcadores de tipo microsatélite, que son secuencias cortas de ADN (uno a cuatro nucleótidos), se repiten un determinado número de veces y se encuentran dispersos dentro del genoma de una especie (Zajc y Sampson, 1999; Curi y Lopes, 2002). Las diferencias en el número de repeticiones constituyen formas alélicas del microsatélite y presentan patrones de herencia mendeliana (Curi y Lopes, 2002). Dentro de las ventajas que ofrecen es que son polimórficos, codominantes, fáciles de medir y analizar, repetitivos y automatizables (Aranguren-Méndez et al., 2005). Se ha recomendado un set de treinta marcadores para su uso en análisis de diversidad genética en bovinos (FAO, 2011).

El perfil obtenido a partir de los marcadores genéticos es utilizado para estudios de diversidad, también es útil para propósitos tales como: identificación individual de animales en análisis forénsico, verificación de parentescos, selección asistida por marcadores genéticos, estimación de consanguinidad, determinación de especies y, más recientemente, en estudios de trazabilidad (Zajc y Sampson, 1999; Curi y Lopes, 2002; Maudet et al., 2002; Aranguren-Méndez et al., 2005; Budowle et al., 2005; Felmer et al., 2006).

En Costa Rica los estudios con marcadores de ADN en ganado bovino son pocos y se han realizado con muestras de tamaño reducido. Un estudio reportó el uso de marcadores genéticos para evaluar diversidad en un hato pequeño de bovinos criollos $(n=12)$, 
Romosinuanos (n=19) y Jersey $(n=10)$ (YañezKemke, 1995). Un segundo estudio se realizó en cincuenta animales de raza no reportada, procedentes de tres diferentes hatos (Navarrete-Barquero, 2005). Otra investigación se realizó en 85 animales de razas Holstein y Jersey, procedentes de cuatro hatos (Fernández-Monge, 2007). Estos estudios, por ser de escala reducida, no reflejan la diversidad genética de la población bovina a nivel nacional. Por ello, el objetivo del presente estudio fue explorar el grado de diversidad genética inter-regional presente en el ganado bovino de Costa Rica.

\section{MATERIALES Y MÉTODOS}

\section{Muestreo}

Se realizó un cálculo preliminar del tamaño mínimo de muestra para medir con precisión adecuada las frecuencias alélicas de los marcadores evaluados. El cálculo se basó en un test de bondad de ajuste chi cuadrado, según el programa GPower v.3.1. (Faul et al., 2007). Entre los parámetros requeridos para el cálculo se consideró el número de regiones por muestrear (8), la cantidad esperada de alelos por marcador (8), el nivel de precisión o tamaño de efecto (0,15 desviaciones estándares), así como un nivel de confianza de $95 \%$ y una potencia estadística de $90 \%$. A partir de estos parámetros se obtuvo un número mínimo de alelos a muestrear de 1809, equivalentes a 905 individuos. Este número se distribuyó de forma proporcional entre las diferentes regiones del país (Cuadro 1, Figura 1), con base en la estructura de la población bovina reportada en el último censo ganadero (CORFOGA, 2000), lo que permitió obtener un estimado de la cantidad mínima de muestras requeridas por región.

El plan de muestreo contempló varios objetivos conjuntos ajenos al presente estudio, por lo que el tamaño y constitución de la muestra final colectada (Cuadro 1, Figura 1) no se ajustó a las proporciones deseadas, pero excedió los mínimos requeridos en casi todas las regiones. Para la selección de los establecimientos a visitar, se utilizó como marco muestral el Sistema Integrado de Registro de Establecimientos Agropecuarios (SIREA) del Servicio Nacional de Salud Animal (SENASA). Con base en las recomendaciones de FAO/ISAG (FAO, 2011) se procuró maximizar el número de establecimientos visitados para evitar muestrear animales emparentados, por lo que se limitó a dos el número de muestras colectadas por establecimiento, salvo en los que se encontraron múltiples tipos raciales.

El número de muestras de pelo fue de 1498, las cuales se colectaron y procesaron durante el año

Cuadro 1. Población bovina (cantidad y porcentual), número mínimo de muestras de ADN requeridas y número final de muestras procesadas exitosamente según región de procedencia. Costa Rica. 2013.

Table 1. Cattle population (number and percentage), minimum number of DNA samples required and the final number of samples successfully processed by region of origin. Costa Rica. 2013.

\begin{tabular}{lcccc}
\hline Región & $\begin{array}{c}\text { Población bovina } \\
\text { (Censo 2000) }\end{array}$ & \% & $\begin{array}{c}\text { Mínimo de muestras } \\
\text { requeridas }\end{array}$ & $\begin{array}{c}\text { Muestras finales } \\
\text { analizadas }\end{array}$ \\
\hline Brunca & 177464 & 13,07 & 118 & 239 \\
Central Metropolitana & 62124 & 4,57 & 41 & 158 \\
Central Occidental & 50619 & 3,73 & 34 & 118 \\
Central Sur & 37709 & 2,78 & 25 & 64 \\
Chorotega & 323503 & 23,81 & 216 & 212 \\
Huetar Caribe & 179579 & 13,22 & 120 & 164 \\
Huetar Norte & 400924 & 29,52 & 267 & 469 \\
Pacífico Central & $\underline{126287}$ & 9,30 & $\underline{84}$ & $\underline{74}$ \\
Total & 1358209 & & 905 & 1498 \\
\hline
\end{tabular}




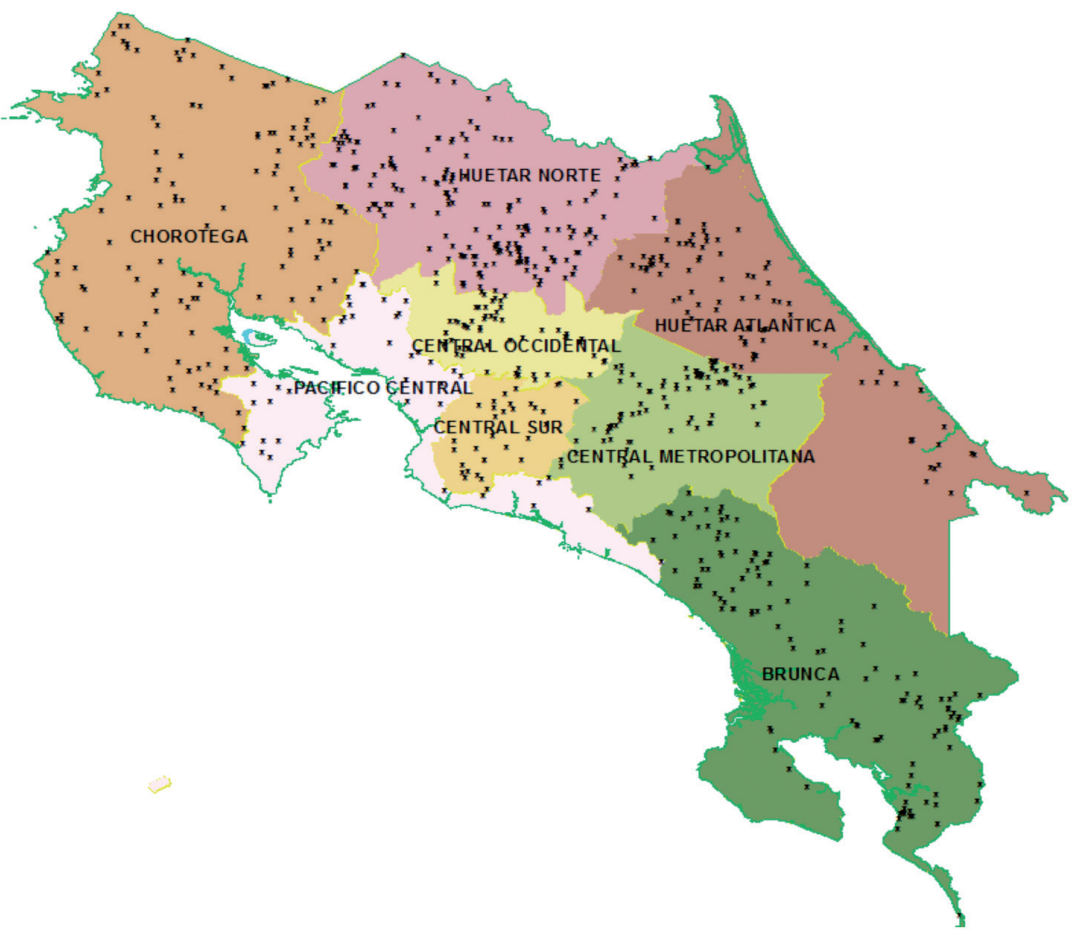

Figura 1. Localización de 1498 muestras de ADN bovino según región de procedencia. Costa Rica. 2013.

Figure 1. Location of 1498 bovine DNA samples according to region of origin. Costa Rica. 2013.

2013. El tamaño varió por región, entre 64 para la región Central Sur y 469 para la región Huetar Norte (Cuadro 1). Para la mayoría de las regiones, excepto la Chorotega y Pacífico Central, se excedió ampliamente el mínimo requerido según el cálculo inicial (Cuadro 1). Se muestrearon un total de 744 establecimientos, de los cuales 243 se dedican a la producción de ganado de carne, 257 al doble propósito y 244 a la producción de leche. La distancia promedio entre puntos de recolección fue de 122,2 $\pm 75,1 \mathrm{~km}$ (Mín=0, Máx= 451).

La recolección de las muestras a nivel de campo se realizó por un equipo de veinticinco funcionarios de las Direcciones Regionales del Servicio Nacional de Salud Animal (SENASA). El material colectado consistió en pelo de la cola, por ser de fácil obtención, manipulación, almacenamiento y preservación a nivel de laboratorio. Mediante pruebas preliminares se determinó que se requería de una muestra de aproximadamente treinta pelos, la cual se arrancó manualmente de la cola del animal debidamente sujetado, realizando un solo movimiento rápido y continuo a contrapelo, de manera que se conservaran los folículos localizados en la raíz. Se requirió además que la muestra estuviera seca, libre de excrementos y orina.

Las muestras se colocaron en sobres individuales en los cuales se indicó la identificación de establecimiento, animal dentro de la unidad productiva, grupo racial estimado por apreciación visual y/o registros, sexo y edad aproximada. Se registró además las coordenadas geográficas del sitio de toma de cada muestra.

\section{Análisis de laboratorio}

Las muestras extraídas de ADN fueron amplificadas por PCR, utilizando un kit con quince de los marcadores internacionales recomendados actualmente por FAO/ISAG (FAO, 2011) para uso 
en bovinos: TGLA227, BM2113, TGLA53, ETH10, SPS115, TGLA126, TGLA122, INRA23, ETH3, ETH225, BM1824, BM1818, CSRM60, CSSM66 y ILSTS006, más otros tres marcadores adicionales: SPS113, RM067 y MGTG4B.

La mezcla de reacción consistió en 1,5 $\mu 1$ de agua grado de biología molecular, $9 \mu 1$ de Mastermix, 9 $\mu 1$ de mezcla de oligos, y $0,5 \mu 1$ de ADN. En todos los casos se incluyó un control positivo (DNA001 incluido en el kit) y un control negativo (agua grado biología molecular). El programa de temperaturas utilizado en el termociclador fue: $98{ }^{\circ} \mathrm{C}$ por $60 \mathrm{~s}, 30$ ciclos de $98^{\circ} \mathrm{C}$ por $20 \mathrm{~s}, 60^{\circ} \mathrm{C}$ por $75 \mathrm{~s}$ y $72^{\circ} \mathrm{C}$ por 30 $\mathrm{s}$, con una extensión final de $72{ }^{\circ} \mathrm{C}$ por $5 \mathrm{~min}$.

Los productos de la amplificación fueron sometidos a electroforesis capilar. Las condiciones de corrida en el analizador genético fueron las establecidas en el módulo GeneScan_36_Pop4, ajustando los siguientes parámetros: $16 \mathrm{~s}$ a $1,0 \mathrm{kV}$ de inyección y $15,0 \mathrm{kV}$ a 60 ${ }^{\circ} \mathrm{C}$ y 1200 s de corrida. Durante la estandarización se procuró que la intensidad de los picos de cada alelo estuviera entre las 1000 y 4000 unidades relativas de fluorescencia (RFUs). Las muestras con picos de alelos menores de 500 y mayores a 6000 fueron analizadas nuevamente, ajustando la concentración de manera que la fluorescencia estuviera en el rango óptimo. Los polimorfismos de los microsatélites fueron discriminados de acuerdo a sus patrones de fluorescencia y tamaño.

\section{Análisis estadístico}

Una vez obtenidos los resultados del procesamiento de muestras de ADN en el laboratorio se procedió a realizar el cálculo de las frecuencias alélicas por marcador, tanto a nivel nacional como por región. Se evaluó el cumplimiento del supuesto de equilibrio Hardy-Weinberg a partir de una prueba exacta basada en cadenas markovianas (100 simulaciones $\times 5000$ iteraciones) según se implementa en el programa Genepop v.4.2.1 (Rousset, 2008). Se calcularon además los siguientes parámetros genéticos poblacionales: número total de alelos $(\mathrm{Na})$, número efectivo de alelos $\left(\mathrm{Ne}=1 / \Sigma p^{2}\right.$, con $\mathrm{p}=$ frecuencias alélicas por marcador microsatélite), la heterocigosis observada (Ho) y esperada $(\mathrm{He})$, el índice de consanguinidad $\left(\mathrm{F}_{\mathrm{IS}}=(\mathrm{He}-\mathrm{Ho}) / \mathrm{He}\right)$ por locus y como promedio para el conjunto de loci evaluados. Estos cálculos se realizaron mediante el programa de cómputo GeneAlex v.6.5 (Peakall y Smouse, 2006, 2012). Se obtuvieron además el contenido de información polimórfica (CIP) y un estimado de la frecuencia de alelos nulos mediante el programa Cervus v.3.03 (Kalinowski et al., 2007).

Se realizó un análisis de varianza molecular (AMOVA) (Excoffier et al., 1992) mediante el programa GeneAlex v6.5 (Peakall y Smouse, 2006, 2012), con el fin de evaluar la existencia de subdivisiones genéticas significativas entre las poblaciones bovinas de las distintas regiones. Mediante este análisis se obtuvieron estimados de las varianzas genéticas entre regiones (Ver), entre individuos (Vei) e intra-individuos (Vii). Esto permitió calcular los estadísticos $\mathrm{F}$ (Wright, 1950), específicamente $F_{S T}=\operatorname{Ver} /(\mathrm{Vii}+\mathrm{Vei}+\mathrm{Ver})$, $F_{I S}=V e i /(V i i+V e i)$ y $\mathrm{F}_{\text {IT }}=(V e i+V e r) /(V i i+V e i+V e r)$. Se obtuvieron estimados de significancia para estos valores F mediante análisis de remuestreo (Felsenstein, 1985) con 1000 permutaciones aleatorias.

Se calcularon las distancias genéticas de $\mathrm{Nei}$ (Nei, 1972) y los estimados de diferenciación genética $R_{S T}$ (Slatkin, 1995) entre todos los pares de regiones mediante GeneAlex v.6.5 (Peakall y Smouse, 2006, 2012). Para $R_{\text {ST }}$ se evaluó la significancia estadística mediante la realización de análisis de remuestreo (Felsenstein, 1985) con 1000 permutaciones aleatorias.

Se construyó un dendrograma con base en las frecuencias alélicas por región mediante el programa Popstree2 (Takezaki et al., 2010) utilizando el procedimiento Neighbor-Joining (Saitou y Nei, 1987). La consistencia de las ramificaciones del dendrograma se evaluó mediante análisis de remuestreo (Felsenstein, 1985) con 1000 permutaciones.

\section{RESULTADOS Y DISCUSIÓN}

\section{Descripción de la muestra}

Las 1498 muestras de ADN analizadas procedieron de una gran diversidad de grupos raciales (Figura 2). Como se especificó previamente, el grupo racial se reportó mayormente con base en la apreciación visual realizada por parte del personal encargado del muestreo. En algunos casos el patrón racial fue evidente porque el fenotipo se ajusta a razas puras ampliamente conocidas, frecuentemente respaldadas por registros genealógicos en el establecimiento (por 


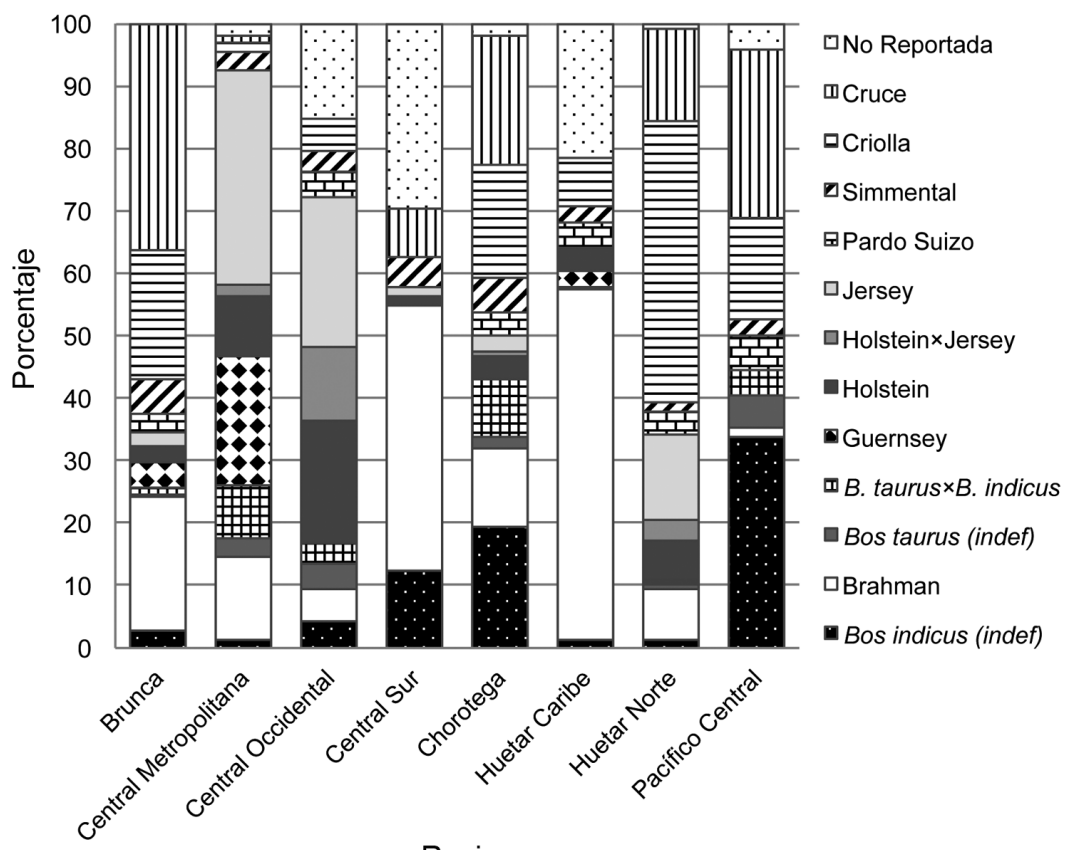

Regiones

Figura 2. Frecuencias relativas absolutas (\%) de muestras de ADN por grupo racial (sectores dentro de columna) según región de procedencia. Costa Rica. 2013.

Figure 2. Absolute relative frequencies (\%) of DNA samples from each racial group (sectors within column) by region of origin. Costa Rica. 2013.

ej. Holstein o Jersey). Sin embargo, en la mayoría de los casos, los bovinos existentes en las fincas eran producto de cruces interraciales, por lo que se hizo difícil establecer con precisión su composición racial.

Se realizó una clasificación simplificada de los grupos raciales de la siguiente manera: Brahman $(n=263)$, Guernsey $(n=49)$, Holstein $(n=89)$, Jersey $(n=158)$, Pardo Suizo $(n=49)$, Simmental $(n=50)$, cruces Holstein $\times$ Jersey $(\mathrm{n}=34)$, múltiples razas minoritarias de tipo Bos indicus (97), múltples razas minoritarias de tipo Bos taurus $(\mathrm{n}=22)$, múltiples cruces minoritarios de tipo Bos taurus $\times$ Bos indicus (43). Otros animales reportados como raza "Criolla" $(n=331)$ o "Cruce" $(n=227)$, mientras que para otros no se reportó raza alguna $(n=86)$. Esta clasificación fue inespecífica en algunos casos, sobre todo para los grupos denominados "Criollo" y "Cruce", los cuales en realidad engloban animales de diversas mezclas raciales. De igual manera, el ganado reportado como Brahman, en algunos casos, correspondía a ganado tipo cebuino que se utilizaba en explotaciones comerciales. Las relaciones entre grupos raciales y frecuencias alélicas deberían ser analizadas en otro estudio complementario.

\section{Análisis nacional}

Al contabilizar las frecuencias alélicas por marcador, se destaca la alta diversidad, ya que todos los marcadores evaluados fueron polimórficos (Cuadro 2). El promedio de alelos observados por marcador fue de 14,6 $\pm 1,01$, variando entre nueve para ETH10 y BM1824 y veinticuatro para TGL122 (Cuadro 2). A pesar del alto número de alelos por marcador, se observó una alta proporción con frecuencias bajas $(<5 \%)$, por lo que el número de efectivos $(\mathrm{Ne})$ fue mucho menor $(5,59 \pm 0,36)$ (Cuadro 3$)$.

El número de alelos observados en el presente estudio fue similar al 13,2 reportado por Egito et al. (2007), pero marcadamente mayor al reportado 
Cuadro 2. Número de alelos observados $(\mathrm{Na})$ y efectivos $(\mathrm{Ne})$, heterocigosis observada (Ho) y esperada (He), contenido de información polimórfica (CIP), índice de consanguinidad $\left(\mathrm{F}_{\mathrm{IS}}\right)$ y prueba de equilibrio Hardy-Weinberg (HW) para los dieciocho marcadores genéticos evaluados en una población bovina nacional. Costa Rica. 2013.

Table 2. Number of observed (Na) and effective (Ne) alleles, observed (Ho) and expected (He) heterozygosity, polymorphic information content (PIC), inbreeding coefficient $\left(\mathrm{F}_{\mathrm{IS}}\right)$ and Hardy-Weinberg equilibrium test $(\mathrm{HW})$ for the eighteen genetic markers analyzed in national cattle population. Costa Rica. 2013.

\begin{tabular}{lccccccc}
\hline Marcador & Na & Ne & Ho & He & CIP $^{\mathbf{f}}$ & $\mathbf{F}_{\text {IS }}$ & HW \\
\hline TGLA227 & 14 & 5,75 & 0,78 & 0,83 & 0,81 & 0,05 & $\mathrm{P}<0,0001$ \\
BM2113 & 11 & 6,99 & 0,82 & 0,86 & 0,84 & 0,04 & $\mathrm{P}<0,0001$ \\
TGLA53 & 19 & 6,29 & 0,78 & 0,84 & 0,83 & 0,07 & $\mathrm{P}<0,0001$ \\
ETH10 & 9 & 6,36 & 0,78 & 0,84 & 0,82 & 0,07 & $\mathrm{P}<0,0001$ \\
SPS115 & 16 & 4,37 & 0,75 & 0,77 & 0,75 & 0,03 & $\mathrm{P}<0,0001$ \\
SPS113 & 21 & 6,99 & 0,80 & 0,86 & 0,84 & 0,06 & $\mathrm{P}<0,0001$ \\
RM067 & 10 & 3,10 & 0,63 & 0,68 & 0,65 & 0,07 & $\mathrm{P}<0,0001$ \\
TGLA126 & 13 & 4,73 & 0,74 & 0,79 & 0,76 & 0,06 & $\mathrm{P}<0,0001$ \\
TGLA122 & 24 & 8,06 & 0,84 & 0,88 & 0,87 & 0,05 & $\mathrm{P}<0,0001$ \\
INRA23 & 15 & 6,03 & 0,80 & 0,83 & 0,82 & 0,04 & $\mathrm{P}<0,05$ \\
BM1818 & 13 & 3,93 & 0,70 & 0,75 & 0,71 & 0,07 & $\mathrm{P}<0,0001$ \\
ETH3 & 13 & 3,80 & 0,70 & 0,74 & 0,71 & 0,06 & $\mathrm{P}<0,0001$ \\
ETH225 & 11 & 5,30 & 0,73 & 0,81 & 0,79 & 0,10 & $\mathrm{P}<0,0001$ \\
BM1824 & 9 & 3,81 & 0,70 & 0,74 & 0,70 & 0,05 & $\mathrm{P}<0,01$ \\
CSRM60 & 18 & 4,57 & 0,75 & 0,78 & 0,75 & 0,04 & $\mathrm{P}<0,0001$ \\
MGTG4B & 13 & 6,53 & 0,83 & 0,85 & 0,83 & 0,02 & $\mathrm{P}<0,05$ \\
CSSM66 & 14 & 8,76 & 0,85 & 0,89 & 0,88 & 0,04 & $\mathrm{P}<0,0001$ \\
ILSTS006 & 20 & 5,27 & 0,76 & 0,81 & 0,79 & 0,07 & $\mathrm{P}<0,0001$ \\
\hline
\end{tabular}

por otros estudios (Maudet et al., 2002; Steigleder et al., 2004; Villalobos-Cortés et al., 2011; Acosta et al., 2013). Puesto que existe coincidencia en una mayoría de los marcadores analizados por los distintos estudios, la mayor cantidad de alelos detectados podría relacionarse con los factores de mayor tamaño de muestra y mayor cantidad de establecimientos visitados. Además, en este estudio se incluyó una alta proporción de animales de grupos raciales cruzados; mientras que la mayoría de los estudios consultados se concentraron en razas específicas.

Los promedios de heterocigosis observada (Ho) y esperada (He) fueron de $0,76 \pm 0,01$ y $0,80 \pm 0,01$, respectivamente. El marcador de menor He fue RM067 con 0,68 y el de mayor He fue CSSM66 con 0,89 (Cuadro 2). El contenido de información polimórfica (CIP) ratificó esta alta variabilidad variando desde un mínimo de 0,65 para el marcador RM067 hasta un máximo de 0,88 para el marcador CSSM66. Estos valores también tendieron a ser mayores a los reportados por estudios similares (Maudet et al., 2002; Steigleder et al., 2004; Pizarro et al., 2009; MartínBurriel et al., 2011; Villalobos-Cortés et al., 2011; Acosta et al., 2013), lo que nuevamente podría ligarse a las características del muestreo, principalmente a la inclusión de mayor diversidad de grupos raciales.

$\mathrm{Al}$ evaluar el supuesto de equilibrio Hardy-Weinberg (HW) para los datos de la población nacional, se encontró que para todos los marcadores hubo evidencia estadística de incumplimiento de dicho supuesto (Cuadro 2). Además, se observó que el valor de $\mathrm{He}$ fue $4 \%$ mayor $(\mathrm{P}<0,001)$ que Ho, lo que implica una deficiencia de heterocigotas. El incumplimiento del equilibrio HW en poblaciones de ganado bovino ha sido reportado en numerosos estudios (Egito et al., 2007; Martín-Burriel et al., 2011; Acosta et al., 2013). La deficiencia de heterocigotas también ha sido reportada en varios estudios con bovinos (Maudet et al., 2002; Egito et al., 2007; Cañón et al., 2008; Martín-Burriel et al., 2011; Acosta et al., 2013). En el presente estudio, el estimado de la 
Cuadro 3. Rango de tamaño en pares de bases (pb) y frecuencias relativas (\%) de los 13 alelos más comunes para cada uno de los 18 marcadores genéticos analizados en la población bovina nacional. Costa Rica. 2013.

Table 3. Size range in base pairs (bp) and relative frequencies (\%) of the 13 most common alleles for each of the 18 genetic markers analyzed in national cattle population. Costa Rica. 2013.

\begin{tabular}{|c|c|c|c|c|c|c|c|c|c|c|c|c|c|c|c|}
\hline \multirow{2}{*}{$\begin{array}{l}\text { Marcador } \\
\text { alélicas }\end{array}$} & \multirow{2}{*}{$\begin{array}{c}\text { Tamaño } \\
\text { (pb) }\end{array}$} & \multicolumn{14}{|c|}{ Frecuencias* } \\
\hline & & 1 & 2 & 3 & 4 & 5 & 6 & 7 & 8 & 9 & 10 & 11 & 12 & 13 & $(\mathrm{~N}<)^{* * *}$ \\
\hline TGLA227 & $77-103$ & 32,9 & 17,2 & 10,1 & 9,5 & 7,2 & 6,9 & 6,4 & 3,7 & 2,9 & 1,2 & 1,1 & 0,5 & 0,3 & (3) \\
\hline BM2113 & $125-145$ & 27,3 & 13,7 & 11,0 & 10,1 & 9,1 & 8,3 & 7,7 & 6,8 & 3,8 & 2,2 & 0,03 & & & - \\
\hline TGLA53 & $154-198$ & 29,1 & 17,7 & 14,0 & 12,5 & 4,1 & 3,8 & 3,6 & 3,4 & 2,4 & 2,1 & 2,0 & 1,5 & 0,9 & (6) \\
\hline ETH10 & $209-225$ & 21,3 & 19,5 & 19,1 & 15,0 & 7,3 & 6,7 & 5,9 & 3,5 & 1,7 & & & & & \\
\hline SPS115 & $244-266$ & 41,7 & 14,4 & 10,5 & 9,0 & 7,5 & 6,9 & 6,5 & 1,9 & 1,1 & 0,2 & 0,1 & 0,1 & 0,03 & (3) \\
\hline SPS113 & $277-301$ & 27,3 & 15,8 & 13,1 & 8,5 & 8,4 & 6,6 & 5,0 & 4,6 & 3,6 & 3,3 & 2,5 & 0,4 & 0,3 & (8) \\
\hline RM067 & 89-108 & 52,3 & 15,4 & 10,9 & 8,5 & 6,5 & 4,4 & 1,5 & 0,4 & 0,1 & 0,03 & & & & - \\
\hline TGLA126 & $105-131$ & 30,0 & 27,4 & 14,4 & 12,7 & 7,8 & 5,5 & 1,3 & 0,3 & 0,2 & 0,2 & 0,1 & 0,1 & 0,03 & - \\
\hline TGLA122 & 137-183 & 23,9 & 17,5 & 10,2 & 9,0 & 7,7 & 6,0 & 5,6 & 4,1 & 4,0 & 2,5 & 2,2 & 1,6 & 1,2 & (11) \\
\hline INRA23 & $194-222$ & 31,8 & 15,5 & 13,2 & 9,9 & 7,8 & 4,5 & 4,2 & 3,8 & 2,9 & 2,8 & 1,8 & 1,3 & 0,4 & (2) \\
\hline BM1818 & $254-286$ & 40,5 & 22,2 & 18,3 & 6,0 & 5,3 & 2,8 & 2,2 & 1,2 & 1,1 & 0,3 & 0,1 & 0,1 & 0,03 & - \\
\hline ETH3 & 103-131 & 45,4 & 16,6 & 11,9 & 8,9 & 5,8 & 4,3 & 3,1 & 2,7 & 0,7 & 0,4 & 0,1 & 0,1 & 0,03 & - \\
\hline ETH225 & $140-162$ & 29,2 & 21,7 & 18,0 & 11,0 & 9,5 & 3,8 & 2,2 & 2,1 & 1,8 & 0,4 & 0,2 & & & - \\
\hline BM1824 & 178-194 & 36,3 & 30,3 & 14,3 & 12,9 & 3,3 & 2,7 & 0,1 & 0,1 & 0,03 & & & & & - \\
\hline CSRM60 & 86-117 & 34,3 & 28,3 & 7,8 & 7,6 & 6,9 & 4,7 & 3,7 & 2,3 & 2,3 & 0,8 & 0,5 & 0,3 & 0,3 & (5) \\
\hline MGTG4B & $129-153$ & 25,7 & 21,4 & 11,0 & 9,8 & 7,9 & 7,6 & 6,7 & 4,2 & 3,4 & 1,2 & 0,8 & 0,2 & 0,1 & - \\
\hline CSSM66 & $179-205$ & 15,3 & 15,0 & 14,4 & 14,3 & 8,9 & 7,6 & 7,2 & 5,7 & 5,5 & 4,4 & 0,9 & 0,5 & 0,3 & (1) \\
\hline ILSTS006 & $282-302$ & 28,2 & 21,9 & 20,0 & 13,7 & 3,4 & 3,2 & 2,2 & 1,9 & 1,2 & 1,1 & 0,7 & 0,7 & 0,6 & (7) \\
\hline
\end{tabular}

* Para cada marcador se muestran un máximo de trece alelos en orden descendente según frecuencias alélicas.

** $(\mathrm{N}<$ ): número de alelos adicionales (no mostrados) con frecuencias menores a los anteriores.

frecuencia promedio de alelos nulos fue 0,03 , variando entre 0,01 para MGTG4B y 0,05 para ETH225. Estos valores se consideraron bajos, por lo que no es un factor determinante en la reducción observada de heterocigosis. En este caso, es más probable que se haya debido a la selección y al control de los apareamientos que realizaron los criadores bovinos, más marcado en las razas puras lecheras como la Holstein o Jersey, donde la tendencia ha sido un uso intensivo de pocos machos reproductores, lo que ha conllevado a incrementos significativos de consanguinidad.

El nivel de endogamia o pérdida de heterocigosis también puede estimarse en función del valor obtenido para $\mathrm{F}_{\text {IS }}$ por cada locus y como promedio sobre el conjunto de loci evaluados (Cuadro 3). El valor promedio fue de $0,06 \pm 0,004$, oscilando entre 0,02 para MGTG4B y 0,10 para ETH225. En ganado bovino, este valor de consanguinidad se considera alto. Los resultados del análisis AMOVA tendieron a respaldar lo mencionado anteriormente, ya que los valores de $\mathrm{F}_{\mathrm{ST}}, \mathrm{F}_{\mathrm{IS}}$ y $\mathrm{F}_{\mathrm{IT}}$ fueron de $0,017,0,044$ y 0,060 , respectivamente, todos altamente significativos $(\mathrm{P}<0,001)$. El valor significativo de $\mathrm{F}_{\mathrm{ST}}$ confirmó que sí existe una diferenciación genética significativa entre las subpoblaciones bovinas de las distintas regiones dentro del país. Se ha cuestionado el uso de estadísticos $\mathrm{F}$, basados en un modelo infinitesimal de mutación, cuando el número de alelos por marcadores es alto, ya que tienden a subestimar el nivel de subestructura poblacional. Por tal motivo, se ha sugerido el cálculo de estadísticos R (Slatkin, 1995) basados en un modelo gradual de mutación. En el presente estudio los valores obtenidos de $\mathrm{R}_{\mathrm{ST}}, \mathrm{R}_{\mathrm{IS}}$ y $\mathrm{R}_{\mathrm{IT}}$ fueron de $0,023,0,051$ y 0,073 , respectivamente, todos altamente significativos $(\mathrm{P}<0,001)$ y ligeramente más altos que los valores $\mathrm{F}$ anteriores.

Los valores reportados de $\mathrm{F}_{\text {IS }}$ en estudios similares en bovinos son altamente variables. Se reportan resultados entre $-0,06$ y 0,29 para cinco razas en 
Cuba (Acosta et al., 2013). Otros valores reportados oscilaron entre $-0,06$ y 0,10 para diecinueve razas ibéricas (Martín-Burriel et al., 2011), entre 0,04 y 0,12 para diez razas en Brasil (Egito et al., 2007), o entre $-0,01$ y 0,25 en una revisión de varios estudios (Villalobos-Cortés et al., 2011). Esta gran variación se puede atribuir a diferencias en los patrones de desarrollo y evolución que han tenido las diferentes razas evaluadas en sus respectivos sitios de procedencia.

\section{Análisis por región}

La variabilidad genética fue similar y alta en todas las regiones del país (Cuadro 4). La heterocigosis observada (Ho) varió desde 0,73 para la región Central Sur hasta 0,78 para la región Huetar Norte. Sin embargo, estos estimados, no difirieron significativamente entre sí $(\mathrm{P}>0,05)$. En todas las regiones se observó una deficiencia de heterocigotas $(\mathrm{Ho}<\mathrm{He})$ que varió entre 2 y $4 \%$, siguiendo el mismo patrón señalado previamente a nivel nacional.

Asimismo, se observó que el índice de consanguinidad $\mathrm{F}_{\text {IS }}$ varió entre 0,03 para las regiones Chorotega, Huetar Caribe y Norte, y 0,06 para las regiones Brunca y Central Sur. Estos fueron menos variables que los reportados por los estudios citados anteriormente (Egito et al., 2007; Martín-Burriel et al., 2011; Villalobos-Cortés et al., 2011; Acosta et al., 2013).

$\mathrm{Al}$ evaluar el supuesto de equilibrio HW dentro de las regiones, existió una mayor tendencia al cumplimiento, siendo que en cinco de las ocho regiones se mantuvo el equilibrio HW para más del $70 \%$ de los loci (Cuadro 4). Por el contrario, las regiones en las que persistió la tendencia al desequilibrio HW fueron la Huetar Norte, la Central Occidental y la Central Metropolitana. Es posible que los menores tamaños de muestra hayan jugado un papel importante, ya que la potencia estadística se reduce y se dificulta declarar desviaciones significativas. Por el contrario, la región con mayor número de muestras (Huetar Norte) fue la que mantuvo la mayor proporción de loci en desequilibrio.

En el dendrograma construido con base en las frecuencias alélicas por región (Figura 3) se observó como las regiones Central Metropolitana y Central Occidental se separaron en la rama superior del árbol. De igual manera, en la rama inferior se separaron las regiones Central Sur, Chorotega, Pacífico Central y Huetar Caribe. Las dos regiones restantes, Brunca y Huetar Norte, se ubicaron en un punto intermedio entre las anteriores. Solo dos de los valores de remuestreo fueron diferentes de 100 , lo que indica que existió fuerte evidencia para separar los tres grupos

Cuadro 4. Número de muestras de ADN procesadas (n), promedio de alelos observados (Na) y efectivos (Ne), heterocigosis observada $(\mathrm{Ho})$ y esperada $(\mathrm{He})$, índice de consanguinidad $\left(\mathrm{F}_{\mathrm{IS}}\right)$ y número de loci con test Hardy-Weinberg significativo $(\mathrm{P} \leq 0,05)$ o no significativo $(\mathrm{P}>0,05)$ según región de procedencia en una población bovina nacional. Costa Rica. 2013.

Table 4. Number of DNA samples processed (n), mean number of observed (Na) and effective (Ne) alleles, observed (Ho) and expected $(\mathrm{He})$ heterozygosity, inbreeding coefficient $\left(\mathrm{F}_{\mathrm{IS}}\right)$ and number of loci with significant $(\mathrm{P} \leq 0.05)$ or not significant $(\mathrm{P}>0.05)$ Hardy-Weinberg test according to the region of origin of a national cattle population. Costa Rica. 2013.

\begin{tabular}{lcccccccc}
\hline Región & $\mathbf{n}$ & $\mathbf{N a}$ & $\mathbf{N e}$ & $\mathbf{H o}$ & $\mathbf{H e}$ & $\mathbf{F}_{\text {IS }}$ & $\begin{array}{c}\text { Loci HW } \\
(\mathbf{P} \leq \mathbf{0 , 0 5})\end{array}$ & $\begin{array}{c}\text { Loci HW } \\
(\mathbf{P}>\mathbf{0 , 0 5})\end{array}$ \\
\hline Brunca & 239 & 12,1 & 5,5 & 0,76 & 0,80 & 0,06 & 5 & 13 \\
Central Metropolitana & 158 & 11,0 & 4,9 & 0,74 & 0,78 & 0,05 & 7 & 11 \\
Central Occidental & 118 & 10,7 & 4,9 & 0,74 & 0,78 & 0,05 & 9 & 9 \\
Central Sur & 64 & 10,1 & 4,9 & 0,73 & 0,77 & 0,06 & 5 & 13 \\
Chorotega & 212 & 12,6 & 5,3 & 0,77 & 0,79 & 0,03 & 2 & 16 \\
Huetar Caribe & 164 & 11,8 & 5,4 & 0,77 & 0,80 & 0,03 & 1 & 17 \\
Huetar Norte & 469 & 13,6 & 5,6 & 0,78 & 0,81 & 0,03 & 11 & 7 \\
Pacífico Central & 74 & 10,2 & 5,1 & 0,75 & 0,79 & 0,05 & 4 & 14 \\
\hline
\end{tabular}




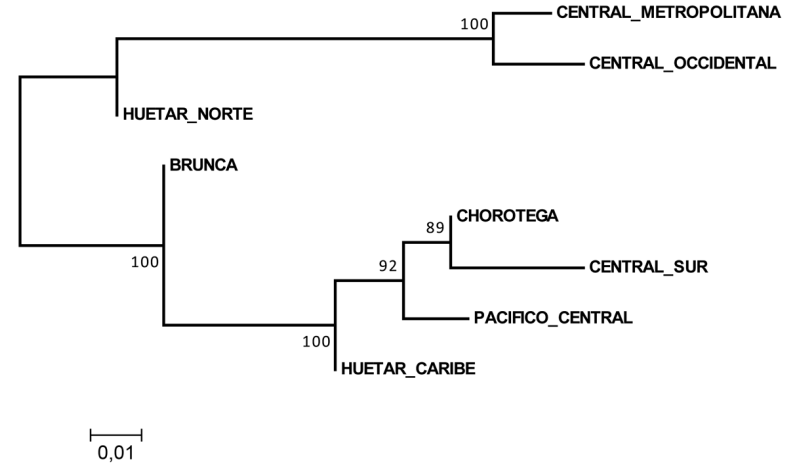

Figura 3. Dendrograma obtenido mediante el método de Neighbor-Joining (Saitou y Nei, 1987) a partir de la matriz de distancias genéticas de Nei (Nei, 1972) entre las ocho regiones analizadas. Costa Rica. 2013.

Figure 3. Dendrogram obtained by the Neighbor-Joining method (Saitou and Nei , 1987) from the matrix of Nei genetic distances (Nei , 1972) among the eight regions analyzed. Costa Rica. 2013.

mencionados anteriormente, y menor evidencia en las últimas dos subdivisiones de la ramificación inferior.

Las distancias genéticas de Nei (1972) (bajo la diagonal, Cuadro 5) variaron entre 0,01 (Chorotega vs.
Huetar Caribe) y 0,27 (Central Occidental vs. Central Sur). Las diferencias $R_{S T}$ entre pares de regiones (Cuadro 5, sobre la diagonal) ratificaron lo observado en el dendrograma, ya que se encontraron diferencias significativas entre las regiones situadas en las tres secciones del árbol (superior, intermedia e inferior), mientras que las diferencias entre regiones dentro de cada sección tendieron a no ser significativas.

Esta estructura fue congruente con lo observado a nivel de campo. En las regiones Central Metropolitana y Central Occidental, además de su proximidad geográfica, existió una predominancia de ganado lechero especializado tipo Bos taurus. Estas regiones son de topografía irregular, de altitud media a elevada, con temperaturas promedio entre 15 y $20^{\circ} \mathrm{C}$, adecuadas para la cría de razas lecheras exóticas como Holstein y Jersey. Por el contrario, en las regiones Central Sur, Chorotega, Pacífico Central y Huetar Caribe, predominaron las razas de carne tipo Bos indicus o los cruces Bos taurus $\times$ Bos indicus. Son regiones más bajas y cercanas a las costas, generalmente de clima cálido/ seco o cálido/húmedo. Por último, las regiones Huetar Norte y Brunca son zonas relativamente extensas localizadas en el extremo norte y sur-este del país, respectivamente, ambas con topografía y condiciones climáticas irregulares, por lo que en ellas se encontró

Cuadro 5. Matriz de distancias genéticas de Nei (bajo diagonal) y estimados $\mathrm{R}_{\mathrm{ST}}$ de diferenciación genética (sobre la diagonal) entre poblaciones bovinas de ocho regiones de Costa Rica. Costa Rica. 2013.

Table 5. Matrix of Nei genetic distances (below diagonal) and $\mathrm{R}_{\mathrm{ST}}$ estimates of genetic differentiation (above the diagonal) among cattle populations of eight regions of Costa Rica. Costa Rica. 2013.

\begin{tabular}{lcccccccc}
\hline & Brunca & $\begin{array}{c}\text { Central } \\
\text { Metropolitana }\end{array}$ & $\begin{array}{c}\text { Central } \\
\text { Occidental }\end{array}$ & $\begin{array}{c}\text { Central } \\
\text { Sur }\end{array}$ & Chorotega & $\begin{array}{c}\text { Huetar } \\
\text { Caribe }\end{array}$ & $\begin{array}{c}\text { Huetar } \\
\text { Norte }\end{array}$ & $\begin{array}{c}\text { Pacífico } \\
\text { Central }\end{array}$ \\
\hline $\begin{array}{l}\text { Brunca } \\
\text { Central }\end{array}$ & 0,09 & $0,03^{* *}$ & $0,03^{* *}$ & $0,03^{* *}$ & $0,01^{* *}$ & $0,00^{*}$ & $0,00^{\mathrm{NS}}$ & $0,01^{*}$ \\
Metropolitana & & $0,00^{\mathrm{NS}}$ & $0,11^{* *}$ & $0,07^{* *}$ & $0,05^{* *}$ & $0,02^{* *}$ & $0,07^{* *}$ \\
$\begin{array}{l}\text { Central Occidental } \\
\text { Central Sur }\end{array}$ & 0,11 & 0,03 & & $0,12^{* *}$ & $0,08^{* *}$ & $0,06^{* *}$ & $0,03^{* *}$ & $0,07^{* *}$ \\
Chorotega & 0,07 & 0,26 & 0,27 & & $0,00^{\mathrm{NS}}$ & $0,01^{*}$ & $0,04^{* *}$ & $0,00^{\mathrm{NS}}$ \\
Huetar Caribe & 0,03 & 0,19 & 0,19 & 0,02 & & $0,00^{\mathrm{NS}}$ & $0,02^{* *}$ & $0,00^{\mathrm{NS}}$ \\
Huetar Norte & 0,02 & 0,14 & 0,15 & 0,04 & 0,01 & & $0,01^{* *}$ & $0,00^{\mathrm{NS}}$ \\
Pacífico Central & 0,02 & 0,05 & 0,05 & 0,10 & 0,05 & 0,04 & & $0,02^{* *}$ \\
\hline
\end{tabular}

NS No significativo $(\mathrm{P}>0,05)$.

* Significativo $(\mathrm{P}<0,05)$.

** Altamente significativo $(\mathrm{P}<0,01)$. 
una población ganadera de mayor diversidad racial, lo que se apreció en la posición intermedia que presentaron en el dendrograma por regiones. Además, la región Huetar Norte poseía un 30\% de la población ganadera del país, lo que sin duda, también contribuyó a que presentase una mayor diversidad genética.

Los resultados obtenidos permitieron obtener un panorama más completo y preciso del grado de diversidad genética presente en el ganado bovino de Costa Rica. Se observó una alta diversidad alélica en la población bovina de Costa Rica, congruente con la alta gama de tipos raciales que se observan a nivel de campo. También se observó evidencia significativa de subdivisión genética de la población, parcialmente ligada a la subdivisión regional, principalmente tres agrupaciones significativamente diferenciadas. Esto fue congruente con el desarrollo histórico y los patrones de diseminación que se han observado para distintos tipos raciales en diferentes zonas geográficas, principalmente en función de las condiciones agroclimáticas y de los sistemas de explotación imperantes.

\section{AGRADECIMIENTO}

Al personal del SENASA que colaboró en la recolección de muestras en las Direcciones Regionales y al procesamiento de las mismas en el Laboratorio de Bioseguridad-LSE.

\section{LITERATURA CITADA}

Aranguren-Méndez, J.A., R. Román-Bravo, W. Isea, Y. Villasmil, y J. Jordana. 2005. Los microsatélites (STR's), marcadores moleculares de ADN por excelencia para programas de conservación: una revisión. Arch. Latinoam. Prod. Anim. 13:30-42.

Acosta, A.C., O. Uffo, A. Sanz, R. Ronda, R. Osta, C. Rodellar, I. Martin-Burriel, and P. Zaragoza. 2013. Genetic diversity and differentiation of five Cuban cattle breeds using 30 microsatellite loci. J. Anim. Breed. Genet. 130:79-86.

Budowle, B., P. Garofano, A. Hellman, M. Ketchum, S. Kanthaswamy, W. Parson, W. van Haeringen, S. Fain, and T. Broad. 2005. Recommendations for animal DNA forensic and identity testing. Int. J. Leg. Med. 119:295-302.
Cañón, J., I. Tupac-Yupanqui, M.A. García-Atance, O. Cortés, D. García, J. Fernández, and S. Dunner. 2008. Genetic variation within the Lidia bovine breed. Anim. Genet. 39:439-445.

CORFOGA (Corporación Ganadera). 2000. Análisis del censo ganadero-2000. http://corfoga.org/2012/wp-content/ uploads/2012/09/censo.pdf (consultado 10 ene. 2014).

Curi, R.A., and C.R. Lopes. 2002. Evaluation of nine microsatellite loci and misidentification paternity frequency in a population of Gyr breed bovines. Braz. J. Vet. Res. Anim. Sci. 39:129-135.

Egito, A.A., S.R. Paiva, M.S. Albuquerque, A. Mariante, L. D’Almeida, S.R. Castro, and D. Grattapaglia. 2007. Microsatellite based genetic diversity and relationships among ten Creole and commercial cattle breeds raised in Brazil. BMC Genet. 8:83.

Excoffier, L., P. Smouse, and J. Quattro. 1992. Analysis of molecular variance for metric distances among DNA haplotypes: application to human mitochondrial DNA restriction data. Genetics 131:479-491.

FAO (Food and Agriculture Organization of the United Nations). 2010. La situación de los recursos zoogenéticos mundiales para la alimentación y la agricultura. http://www.fao.org/docrep/012/a1250s/ a1250s.pdf (consultado 15 nov. 2014).

FAO (Food and Agriculture Organization of the United Nations). 2011. Molecular genetic characterization of animal genetic resources. FAO Animal Production and Health Guidelines 9. Rome, ITA.

Faul, F., E. Erdfelder, A.G. Lang, and A. Buchner. 2007. G*Power 3: A flexible statistical power analysis program for the social, behavioral, and biomedical sciences. Behav. Res. 39:175-191.

Felmer, R., R. Chávez, A. Catrileo, y C. Rojas. 2006. Tecnologías actuales y emergentes para la identificación animal y su aplicación en la trazabilidad animal. Instituto de Investigaciones Agropecuarias. Ach. Med. Vet. 3:197-206.

Felsenstein, J. 1985. Confidence limits on phylogenies: An approach using the bootstrap. Evol. 39:783-791.

Fernández-Monge, M. 2007. Determinación de las frecuencias alélicas de 11 marcadores microsatélites en dos razas de ganado lechero en Costa Rica. Tesis Bach. Biotecnología, Escuela de Biología, Instituto Tecnológico de Costa Rica, CRC.

Kalinowski, S.T., M.L. Taper, and T.C. Marshall. 2007. Revising how the computer program CERVUS accommodates genotyping error increases success in paternity assignment. Mol. Ecol. 16:1099-1006. 
Lara, M.A.C., E.P.B. Contel, y J.R.B. Sereno. 2005. Caracterización genética de poblaciones cebuínas a través de marcadores moleculares. Arch. Zootec. 54:295-303.

Martín-Burriel, I., C. Rodellar, J. Cañón, O. Cortés, S. Dunner, V. Landi, A. Martínez-Martínez, L.T. Gama, C. Ginja, M.C.T. Penedo, A. Sanz, P. Zaragoza, and J.V. Delgado. 2011. Genetic diversity, structure, and breed relationships in Iberian cattle. J. Anim. Sci 89:893-906

Maudet, C., G. Luikart, and P. Taberlet. 2002. Genetic diversity and assignment tests among seven French cattle breeds based on microsatellite DNA analysis. J. Anim. Sci. 80:942-950.

Navarrete-Barquero, D. 2005. Determinación de las frecuencias alélicas de 11 marcadores microsatélites en el ganado bovino de engorde de Costa Rica. Tesis Bach. Biotecnología, Escuela de Biología, Instituto Tecnológico de Costa Rica, CRC.

Nei, M. 1972. Genetic distance between populations. Am. Nat. 106:283-292.

Peakall, R., and P.E. Smouse. 2006. GenAlEx 6: genetic analysis in Excel. Population genetic software for teaching and research. Mol. Ecol. 6:288-295.

Peakall, R., and P.E. Smouse. 2012. GenAlEx 6.5: genetic analysis in Excel. Population genetic software for teaching and research - an update. Bioinformatics 28:2537-2539.

Pizarro, M.G., F. Mujica, y R. Felmer. 2009. Estructura poblacional y diversidad genética de rebaños bovinos de carne del sur de Chile. Agro Sur 37:60-83.

Quirós, E. 2006. Historia de la ganadería en Costa Rica. http://www.corfoga.org/images/public/documentos/ pdf/Historia_Ganaderia_bovina.pdf (consultado 10 ene. 2014).
Rousset, F. 2008. Genepop'007: a complete reimplementation of the genepop software for Windows and Linux. Mol. Ecol. Resour. 8:103-106.

Saitou, N., and M. Nei. 1987. The neighbor-joining method: A new method for reconstructing phylogenetic trees. Mol. Biol. Evol. 4:406-425.

Slatkin, M.A. 1995. Measure of population subdivision based on microsatellite allele frequencies. Genetics 139:457-462.

Steigleder, C.S., E.A. Almeida, and T.A. Weimert. 2004 Genetic diversity of a brazilian creole cattle based on fourteen microsatellite loci. Arch. Zootec. 53:3-11.

Takezaki, N., M. Nei, and K. Tamura. 2010. POPTREE2: Software for constructing population trees from allele frequency data and computing other population statistics with Windows interface, Mol. Biol. Evol. 27:747-752.

Villalobos-Cortés, A.I., A.M. Martínez, J.L. Vega-Pla, y J.V. Delgado. 2011. Estructura genética y cuello de botella de la población bovina guaymí mediante microsatélites. Arch. Zootec. 60:767-775.

Wright, S. 1950. Genetical structure of populations. Nature 166:247-249.

Yañez-Kemke, M.A. 1995. Uso de marcadores moleculares RAPDs (Random amplified polimorphic DNA) en ganado bovino adaptado a condiciones de trópico húmedo. Tesis Mag. Sc. Centro Agronómico Tropical para la Investigación y Enseñanza (CATIE), Turrialba, CRC.

Zajc, I., and J. Sampson. 1999. Utility of canine microsatellites in revealing the relationships of pure bred dogs. J. Hered. 90:104-107. 